\title{
LA INFLUENCIA DEL TIEMPO ESCOLAR EN EL RENDIMIENTO ACADÉMICO: UN ESTUDIO COMPARATIVO EN SISTEMAS EDUCATIVOS DE EUROPA Y LATINOAMÉRICA
}

\author{
The influence of school time in the academic achievement: a comparative study \\ in educational systems of Europe and Latin America
}

\author{
Ernesto Colomo Magaña \\ Andrea Cívico Ariza \\ Vicente Gabarda Méndez. \\ Nuria Cuevas Monzonís
}

Fecha recepción: 10/07/2016

Fecha aceptación: 09/11/2016

RESUMEN: Los sistemas educativos están estructurados a tenor de múltiples factores que lo condicionan y determinan. Entre los diferentes aspectos, el tiempo juega un papel clave al marcar la duración de cada una de las diferentes etapas educativas y del proceso de enseñanza-aprendizaje durante las mismas. Partiendo de esta realidad, este trabajo pretende analizar el tiempo escolar y su influencia en el rendimiento académico, considerándolo un factor clave de la calidad educativa. Para ello, se ha llevado a cabo un estudio comparativo entre varios países europeos (Finlandia, Austria, Francia y España) y latinoamericanos (Chile, México y Colombia) que han participado en el Programa para la Evaluación Internacional de los Alumnos (PISA). A partir de los resultados académicos de PISA, pretendemos analizar cómo influyen las horas de instrucción obligatoria (Primaria y Secundaria) en el rendimiento académico del alumnado. Entre los resultados obtenidos, podemos destacar que el simple incremento de la carga horaria en las diferentes etapas educativas no influye positivamente en el rendimiento académico de los alumnos. Por el contrario, cobra mayor relevancia la forma de abordar el proceso de enseñanza-aprendizaje (lo que hacemos como profesionales de la educación en el aula) que el tiempo que se emplee en ello.

PALABRAS CLAVES: Política de la Educación, Educación Comparada, Tiempo Lectivo, Enseñanza Primaria, Enseñanza Secundaria.

ABSTRACT: Educational systems are structured under multiple factors that condition and determine. Between different aspects, the time plays a key role to set the duration of each of the different educational stages and the teaching-learning process for them. Starting from this reality, this paper analyzes the school time and its influence on the academic performance, as one of the main factors in the quality of education. For this, it has been carried out a comparative study of several European countries (Finland, Austria, France and Spain) and Latin American countries (Chile, Mexico and Colombia) have participated in the Programme for International Student Assessment (PISA). From the academic results of PISA, we analyze how influences the hours of compulsory education (Primary and Secondary) in student performance. Between the results obtained, we can stand out that simply increasing the number of hours in the different educational stages no positive effect in student 
performance. Inversely, becomes more important the way of approach the teaching-learning process (whatever we as professional education in the classroom) that the time used in it.

KEYWORDS: Educational Policy, Comparative Education, Teaching Time, Primary education, Secondary education

\section{Introducción}

El hombre vive para alcanzar la plenitud de la vida en la brevedad del tiempo (Savater, 1999, p.276).

El presente estudio pretende abordar el tiempo escolar como factor que define e influye en la calidad de la educación, entendiendo la misma como el éxito respecto a los resultados académicos. Siendo conscientes de que los resultados académicos representan una variable de carácter multifactorial, donde intervienen factores de carácter personal, social, económico y educativo, centraremos nuestra atención en el tiempo escolar como elemento vertebrador de los procesos de enseñanza y aprendizaje.

El tiempo es una de las variables más importantes para abordar el aprendizaje escolar, al ser una dimensión y competencia docente clave en cualquier contexto de enseñanzaaprendizaje (Fernández, 2008). Consideramos este factor como elemento de análisis por ser un componente básico y común en cualquier sistema educativo, considerando, además, que adquiere una relevancia distinta en función del contexto geográfico, social, cultural, político y económico de los diferentes sistemas educativos que conformarán nuestra muestra de estudio. Somos conscientes, como apunta Prats (2013, citado por de Oliveira y Castioni, 2016), que los modos en que se organizan los tiempos lectivos difieren y poseen una amplia variabilidad dentro de los países que conforman la muestra. Además, en los procesos de enseñanzaaprendizaje hay que distinguir entre el tiempo de clase y el tiempo lectivo útil, el cual suele ser bastante menor debido a los diferentes agentes de desconcentración (conductas disruptivas) y obligaciones burocráticas que pueden estar presentes en el aula (completar los partes de incidencias, por ejemplo). Jabonero (2015), nos expone el caso concreto del tiempo útil lectivo en Chile, donde un estudio del Banco Mundial (Burns y Luque, 2014), verificó como los docentes chilenos dedican menos del $65 \%$ del tiempo lectivo al proceso educativo, quedando más de la tercera parte $(35 \%)$ destinado a otras actividades, como resolver conflictos, poner orden o pasar lista.

No obstante, para el estudio de esta variable en nuestro trabajo, profundizaremos en cómo pueden influir aspectos como la duración de la jornada, su distribución y el calendario escolar en los resultados de los estudiantes de la etapa de primaria y secundaria, dejando el aspecto del aprovechamiento real de tiempo lectivo para futuras investigaciones.

El análisis se centrará en una muestra conformada por cuatro países europeos (Finlandia, Austria, Francia y España) y tres países latinoamericanos (Chile, México y Colombia). El motivo de la elección de estos países es su participación en el Programa para la Evaluación Internacional de los Alumnos (PISA) desarrollado en los países que conforman la Organización para la Cooperación y el Desarrollo Económico (OCDE) y algunos países invitados (como es el caso de Colombia). Además, los países seleccionados muestran diferencias notables en la estructuración temporal de sus sistemas educativos, por lo que analizar propuestas distintas a tenor de los resultados académicos obtenidos, nos permitirá extraer algunas conclusiones sobre cuáles pueden ser las medidas más favorables y eficaces a tener en cuenta para realizar la distribución de las horas de instrucción obligatoria. 
Realizaremos este estudio utilizando como metodología/estrategia el método comparativo, ya que nos permitirá obtener una perspectiva más global de la realidad de los distintos contextos escolares. Teniendo como punto de partida las ideas propuestas por Manzón (2010), partimos de unidades supranacionales para la comparación (representada por los países de la Unión Europea y Latinoamérica). La comparación nos permitirá conocer alternativas sobre cómo habría que gestionar el factor tiempo en pos de mejorar la calidad de los procesos de enseñanza-aprendizaje, pues observando se aprende y comparando se crece (Lázaro, 2012).

Tanto el análisis como las conclusiones o propuestas que realicemos, se basan únicamente en los datos relativos a dicho indicador y su relación con los resultados académicos obtenidos en PISA, utilizando la posición relativa de los países como forma de expresar los resultados y medir el rendimiento educativo (Froemel, 2006). Sin duda, el tiempo es un elemento fundamental en el proceso de desarrollo de los seres humanos, por lo que su impacto y gestión durante el proceso educativo repercutirá de forma notable en los progresos y evoluciones de los discentes. Nuestro propósito, que se convertirá en el objetivo principal de este estudio, es conocer hasta qué punto puede la organización y distribución del tiempo influir o determinar los resultados académicos a nivel educativo.

Cabe destacar que trabajaremos desde la perspectiva del constructivismo para la realización de este estudio. La elección de este paradigma constructivista/interpretativo se debe a que el conocimiento que aportemos será una interpretación de la realidad contextual (los datos y resultados comparados entre los países que conforman nuestra muestra) de la forma más objetiva posible, estando siempre abiertos al debate, la reflexión y la reconstrucción de los mismos (Colomo, 2013).

\section{Fundamentación teórica. El informe PISA como exponente del éxito académico}

El informe PISA (su nombre proviene de las siglas del Programa para la Evaluación Internacional de Alumnos en inglés "Programme for International Student Assessment") es un proyecto de la OCDE cuyo fin es medir los resultados académicos de los alumnos al final de la etapa de enseñanza obligatoria. La elección de los escolares al final de la escolarización obligatoria (15 años) los convierte en un grupo adecuado para valorar su grado de preparación para afrontar los desafíos de nuestras sociedades actuales (Turner, 2006).

El objetivo de PISA es ofrecer información para que los países puedan tomar las decisiones y políticas adecuadas para mejorar sus procesos de enseñanza-aprendizaje. Sin embargo, tiene el inconveniente de no tener en consideración los contextos de los países participantes ni las particularidades de cada uno de los sistemas educativos. Los exámenes son fruto de la colaboración entre los países que han de aplicarlos, buscando así la universalización de su contenido. En PISA se evalúan las áreas de lectura, matemáticas y competencia científica mediante preguntas estandarizadas para todos los países por igual, clasificándolos posteriormente según sus resultados.

Desde esta perspectiva, la calidad educativa está sujeta a unos resultados académicos estandarizados para una confluencia de países con contextos sociopolíticos y sistemas educativos diferentes, siendo el factor determinante de esa calidad la puntuación y posición que se ocupe respecto al resto de participantes. Debemos tener claro que PISA, por su universalización y estandarización, está diseñado para reconocer y valorar las destrezas y conocimientos de los alumnos, y no el desempeño de los docentes o del sistema educativo vigente (Bottani, 2006). Sobre esta misma idea también hay que señalar, como recoge 
Hernández (2006), que la escuela secundaria tiende a enseñar para la reproducción, mientras que las pruebas PISA están dirigidas a comprobar las capacidades de transferencia de conocimientos a situaciones cotidianas.

No obstante, los datos que nos ofrece PISA nos permiten tener una base para realizar estudios, investigaciones y análisis orientados a la toma de decisiones respecto a los diferentes indicadores y variables que influyen en la calidad de la educación, centrada en los resultados del aprendizaje (Velaz de Medrano, 2006). Como sostiene Schleicher (2006), "la información facilitada por PISA debería permitir a los responsables políticos observar qué factores están asociados al éxito educativo" (p. 23). Así, cada 3 años los países que participan en estas pruebas pueden valorar cómo han podido influir los objetivos que se habían propuesto mejorar desde la anterior aplicación, extrayendo conclusiones sobre la eficacia y funcionalidad de sus medidas.

Figura 1. Países de la muestra clasificados por orden descendente según sus puntuaciones medias en las distintas disciplinas evaluadas por PISA 2012.

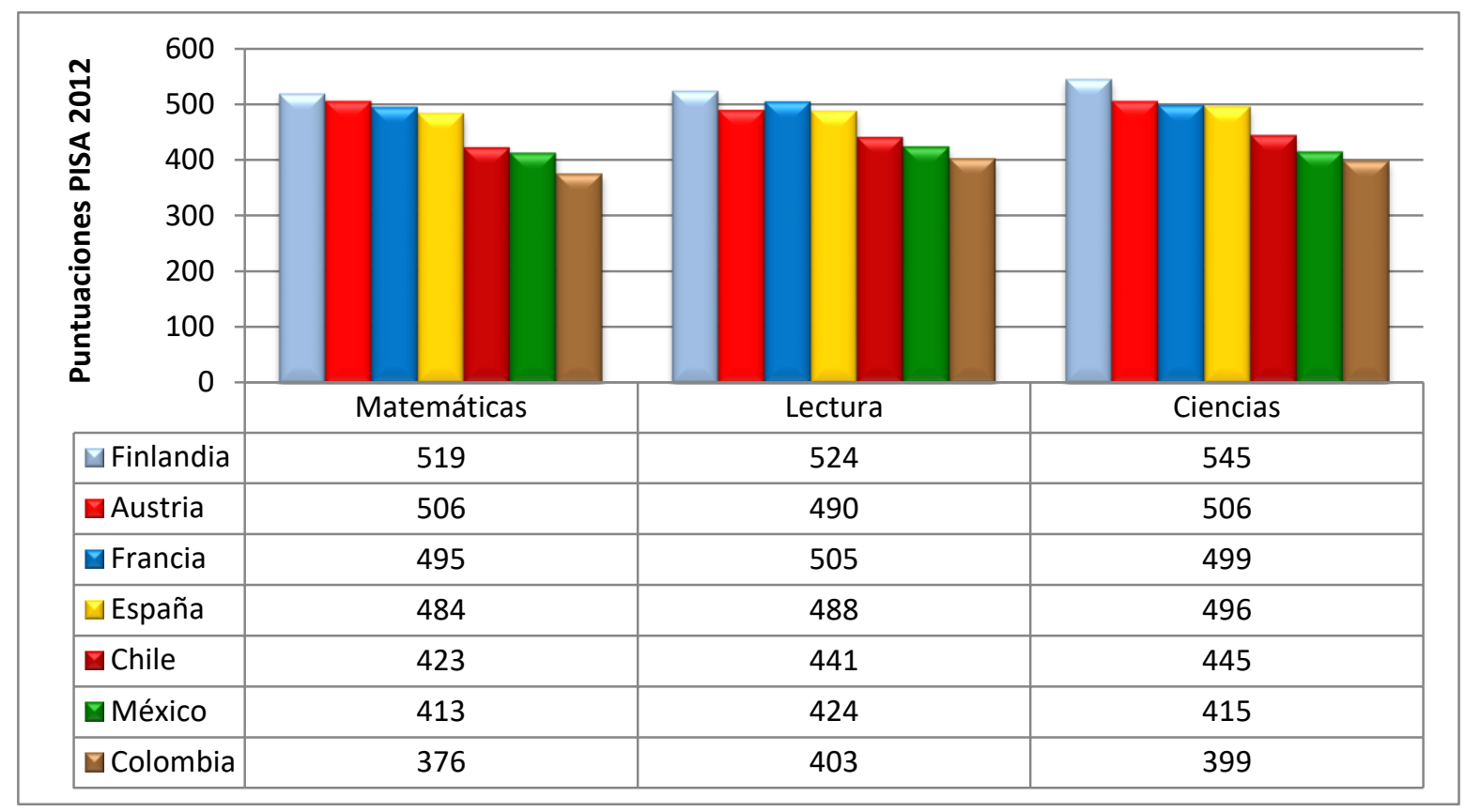

Fuente: Elaboración propia a partir de OCDE (2013).

Asociando la calidad del proceso educativo a los resultados académicos obtenidos, somos conscientes que éstos dependen de la interrelación de otras muchas variables como son el contexto, las políticas y sistemas educativos, la economía, la formación del profesorado y su relación con los discentes, el tiempo escolar, etcétera. PISA nos ofrece la posibilidad de realizar comparaciones internacionales del rendimiento de los alumnos, analizando en profundidad los resultados obtenidos, que son reflejos de la eficacia y eficiencia de los procesos educativos. A partir de los datos, podremos comprender en profundidad los objetivos, fortalezas y oportunidades que dicho estudio ofrece para diseñar propuestas que puedan mejorar los resultados, atendiendo a los diferentes factores que influyen el éxito educativo.

A continuación, profundizaremos en la metodología que vamos a emplear durante nuestro trabajo. 


\section{Metodología}

Como sostiene Colomo (2015), entendemos la metodología como una guía que nos lleva hasta el fin que perseguimos y deseamos, por lo que a la hora de plantearnos el diseño metodológico debemos tener clara la intención que pretendemos alcanzar con nuestro estudio, debido a que diferentes intenciones necesitarán, por consiguiente, diferentes estrategias metodológicas.

Por ello, para alcanzar los fines perseguidos con este estudio, partiremos del método comparativo clásico, impulsado por autores como Vexliard, Bereday o Hilker entre otros (Lázaro, 2012). Roselló (1963), entendía el método comparativo como "la aplicación de la técnica de la comparación al estudio de determinados aspectos de los problemas educativos" (p. 13), siendo variado su ámbito de aplicación (sistemas educativos, planes y programas formativos, métodos y estructuras de enseñanza, etc.). Hemos de destacar las ideas de Vexliard (1970), quien considera que la comparación consiste en:

"la comparación consiste en descubrir y analizar las similitudes y diferencias entre distintos sistemas de educación, entendiendo por esto la organización, la estructura y la administración de la enseñanza, así como también los programas, los métodos pedagógicos, didácticos y de control que se usan en los diversos niveles y especialidades de la enseñanza" (p. 18).

En nuestro estudio, partiendo desde la perspectiva comparativa (Gabarda, 2015), trabajaremos sobre la influencia del tiempo en la calidad educativa a partir de los resultados académicos obtenidos por los países participantes en las pruebas PISA.

En la fase descriptiva, haremos una recopilación de todos los datos recogidos por los informes en materia educativa de la OCDE, en los últimos años, sobre el panorama de la educación a partir de los indicadores investigados por esta organización internacional. El tiempo escolar como uno de los Indicadores de Sistemas Educativos (INES) será nuestra variable, la cual compararemos para poder interpretarla correctamente mediante un análisis pormenorizado. De este modo, los informes de la OCDE serán la fuente principal para la realización de nuestro estudio comparativo, junto con los resultados del informe PISA. El análisis de las horas de instrucción hará referencia a la etapa de escolaridad obligatoria de Educación Primaria y de Educación Secundaria Inferior. Para el mismo, hemos tomado en consideración las reflexiones de Bray y Kai (2010) respecto a la comparación de sistemas educativos, tomando éstos como un elemento dentro de las identidades nacionales de unidades comparativas más amplias, los propios estados.

Entendemos que los resultados de nuestro estudio, siguiendo las ideas de Ruíz Román (2004), son una interpretación fundamentada y abierta a la crítica, sobre la correlación entre la variable tiempo escolar y resultados académicos de los países que hemos tomado como muestra. Todo el conocimiento que aportemos va a ser relativo a las interpretaciones sobre los datos trabajados, ya que como afirma el profesor Pérez Gómez (1998), "el propio conocimiento en ciencias sociales no puede ser más que una peculiar interpretación, sin duda más contrastada y mejor elaborada y argumentada, pero en definitiva una interpretación provisional y parcial de una realidad también efímera" (p.61).

Como recogimos en la introducción, partimos del construccionismo, entendiendo de que no hay una única interpretación sobre la influencia del tiempo escolar en el rendimiento académico, sino que esta depende también de otros factores y variables (contexto, política, economía, sociedad, etc.) que pueden dar lugar a diferentes explicaciones dentro del mismo fenómeno (Sandín, 2003). 
Por lo tanto, las unidades de comparación asociadas a la variable tiempo escolar y sus análisis, quedarán abiertas para las modificaciones que se estimen oportunas en función de las diferentes perspectiva y enfoques desde los cuales se puede abordar el estudio.

\section{Análisis. La influencia del tiempo escolar en los resultados educativos}

Presentamos a continuación los principales resultados obtenidos sobre cómo influye la variable tiempo escolar en el rendimiento académicos de los educandos. De esta manera, podremos analizar cómo influyen aspectos como el número de cursos escolares por etapas, los días lectivos en los mismos, el número de horas de instrucción obligatoria por cada curso, o cómo se distribuyen dichas horas y marcan la duración de las jornadas escolares en las etapas de educación primaria y secundaria.

Para ello, pasamos a analizar las horas de enseñanza de instrucción obligatoria.

\subsection{Horas de enseñanza de instrucción obligatoria}

Dentro de los períodos en los que los alumnos asisten de manera obligatoria a las instituciones educativas, es muy importante analizar el tiempo que pasan dentro de las mismas. No existe homogeneidad en la distribución de la carga horaria en los países analizados, como puede observarse en la siguiente tabla, donde cada uno apuesta por una fórmula distinta respecto al número de días, horas por días e incluso años.

Tabla 1. Datos referentes a las horas de enseñanza de la instrucción obligatoria (Primaria ${ }^{1}$ y Secundaria ${ }^{2}$ ).

\begin{tabular}{|l|l|l|l|l|l|l|l|l|l|l|l|l|l|}
\hline \multirow{2}{*}{ Países } & \multicolumn{3}{|c|}{$N^{\circ}$ Cursos } & \multicolumn{3}{c|}{$N^{\text {o días }}$} & \multicolumn{3}{c|}{ Horas/año } & \multicolumn{3}{c|}{ Totales } \\
\cline { 2 - 13 } & rim. & ec. & otal & rim. & ec. & otal & rim. & ec. & edia & rim. & ec. & otal \\
\hline Finlandia & & & & 87 & 87 & 74 & 32 & 44 & 03 & 794 & 533 & 327 \\
\hline Austria & & & & 80 & 80 & 60 & 05 & 00 & 02 & 820 & 600 & 420 \\
\hline Francia & & & & 62 & 80 & 42 & 64 & 91 & 20 & 320 & 964 & 284 \\
\hline España & & & 0 & 75 & 75 & 50 & 87 & 061 & 97 & 725 & 245 & 970 \\
\hline Chile & & & & 90 & 90 & 80 & 049 & 062 & 052 & 293 & 123 & 416 \\
\hline México & & & & 00 & 00 & 00 & 00 & 167 & 22 & 800 & 500 & 300 \\
\hline Colombia & & & & 00 & 00 & 00 & 000 & 200 & 088 & 000 & 800 & 800 \\
\hline
\end{tabular}

Fuente: Elaboración propia a partir de OCDE (2015).

\footnotetext{
${ }^{1}$ La etapa de instrucción obligatoria de Primaria aparecerá en la tabla con la abreviatura Prim.

${ }^{2}$ La etapa de instrucción obligatoria de Secundaria aparecerá en la tabla con la abreviatura Sec.
} 
Es importante analizar e interpretar los datos referidos al número de horas totales de instrucción obligatoria (etapas de Primaria y Secundaria) de los países que componen la muestra objeto de estudio para poder comprender como afecta esta variable al rendimiento académico. Finlandia destaca por ser el país con el menor número de horas de instrucción obligatoria (6327 horas) y ser la que mejores resultados obtiene dentro de la selección de países de estudio. Este país ha sido y es un referente mundial a nivel educativo, donde la consigna de "menos es más" que defienden y promulgan encuentra su argumentación en varios factores, entre ellos el del tiempo escolar. Tomando como referente sus datos, y comparándolos con los del resto de países que estamos analizando, encontramos que:

- Austria obtiene menor puntuación (13 en matemáticas, 34 en lectura y 39 en ciencias), pese a tener prácticamente la misma carga horaria (73 horas más Austria). No obstante, es el segundo país en la clasificación respecto a los resultados y también tiene un menor número de horas de instrucción obligatoria en proporción al resto de la muestra, lo que denota que el caso de Finlandia con menor tiempo escolar y buenos resultados no es solo una excepción, sino que puede ser factible siempre que adaptemos las propuestas a los contextos pertinentes. También queremos reseñar como sus pequeñas diferencias respecto a la carga horaria total no se debe a una distribución similar en lo que respecta a los cursos académicos. Austria sostiene la fórmula 4+4, con 180 días por cada etapa (suman 14 días menos en total que Finlandia entre las 2 etapas), mientras que Finlandia aboga por $6+3$, teniendo de partida un año más de instrucción obligatoria. De esta manera, es el número de horas por día en las diferentes etapas lo que consigue asemejar el resultado del número de horas totales entre ambos países, teniendo Finlandia un registro horario inferior en las jornadas escolares tanto de primara como de secundaria.

- Países como Francia y España (dentro del marco europeo), con cargas horarias totales considerablemente superiores (31\% superior en Francia y $42 \%$ en España), obtienen resultados por debajo de Finlandia y Austria (salvo Francia en la competencia de lectura que obtiene 505 puntos). No obstante, Francia obtiene mejores resultados que España (11 puntos en matemáticas, 17 en lectura y 3 en ciencias) con un número de horas totales de instrucción obligatoria menor (8284 horas en Francia por las 8970 en España, un total de 686 horas menos).

- Los países latinoamericanos son los que obtienen los resultados académicos más bajos del informe PISA (con diferencias de 96 (Chile), 106 (México) y 143 Colombia) puntos en matemáticas; de 83 (Chile), 100 (México) y 121 (Colombia) puntos en lectura; y de 100(Chile), 130 (México) y 146 (Colombia) en ciencias) pese a tener una mayor carga horaria de instrucción obligatoria (33\% superior en Chile con un total de 8416 horas, $31 \%$ en México con un total de 8300 horas y 55\% en Colombia con un total de 9800). Dentro de los resultados de los países latinoamericanos, destacar como solo en el caso de Chile respecto a México, el mayor número de horas de instrucción obligatoria entre las dos etapas si va asociado a unos mejores resultados. Chile tiene 416 horas más (aunque tiene un curso escolar menos (8 en Chile por 9 en México)) de instrucción obligatoria que México, aventajándola en las puntuaciones de PISA (10 en Matemáticas, 17 en Lectura y 30 en Ciencias).

En este caso concreto, salvando la excepción en Latinoamérica respecto a la comparativa entre Chile y México, podemos rechazar a partir de los datos expuestos tanto la tesis de Schiefelbein, Vélez y Valenzuela (1997), quienes sostenían que existía una correlación positiva entre el número de horas de instrucción y un mayor rendimiento académico; como la más actual de Lavy (2015), que afirma que un mayor número de horas es 
positivo para el desempeño y los resultados de los estudiante. Como podemos ver en nuestro estudio, el aumento de las horas lectivas sin considerar otros factores interdependientes como son los curriculares, pedagógicos o metodológicos, no conlleva un mejor rendimiento académico. De esta manera podemos manifestar que, dentro de los factores que determinan la calidad a nivel escolar, el simple incremento del tiempo escolar no conlleva una mejora en el rendimiento educativo. La calidad, respecto a esta variable, no reside en el indicador cuántas horas estamos enseñando, sino en qué hacemos durante esas horas en el proceso de enseñanza-aprendizaje, pues se ha comprobado que sistemas educativos (Finlandia y Austria) con menor carga horaria de instrucción obligatoria obtienen mejores resultados, siendo más relevante la calidad educativa por encima de la cantidad.

Siguiendo con el análisis del factor tiempo, observando los datos podemos extraer otra interpretación relevante. Se aprecia un mayor índice de rendimiento académico (traducido en mejores puntuaciones medias en los resultados del informe PISA), en función de la relación establecida entre las variables "Número de días" y "Horas/año".

Tras hacer la media de cada uno de los países que conforman nuestro estudio, hemos podido observar cómo el rendimiento académico (resultados) es mayor cuando las horas lectivas de la jornada escolar son menores.

Figura 2. Media de horas diarias de enseñanza durante la instrucción obligatoria (Primaria y Secundaria).

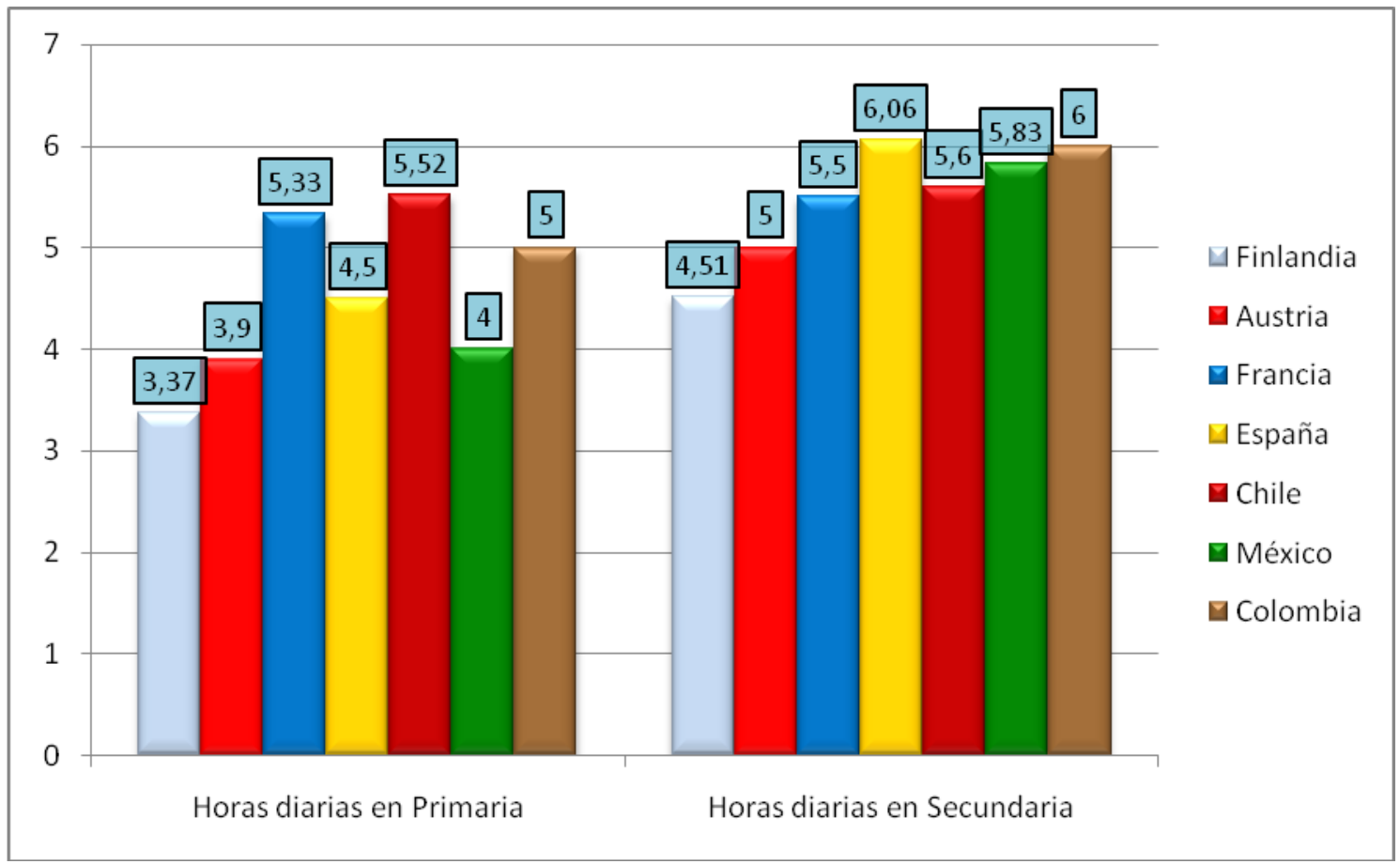

Fuente: Elaboración propia a partir de OCDE (2013).

De esta manera, Finlandia y Austria muestran los mejores resultados teniendo menos horas al día tanto en primaria (jornada lectiva inferior a 4 horas) como en secundaria (en este caso, inferior o igual a 5 horas). No obstante, pese a esta similitud en la menor duración de la jornada lectiva, Finlandia obtiene mejores resultados teniendo un mayor número de días lectivos (7 en ambos niveles) pero con jornadas escolares menores que en el caso de Austria. En el resto de países, en primaria la jornada escolar supera las 4 horas, siendo las más largas 
las de Colombia (5 horas), Francia (5,33 horas) y Chile (5,52 horas); y en secundaria las 5 horas, teniendo a España (6,06 horas) y Colombia (6 horas) como los países con mayor carga horaria. De este modo, podemos observar cómo un mayor número de horas por jornada escolar no se traduce en mejores resultados. Queremos destacar el caso concreto de Colombia, cuya organización temporal le hace ser el país de la muestra con la mayor carga horaria de instrucción obligatoria (9800 horas), teniendo una de las jornadas escolares más largas en ambos niveles y, sin embargo, es el país con los peores resultados de todos los que hemos trabajado. Por el contrario, un menor número de horas lectivas por día reportan mayores resultados.

Partiendo de estos datos podemos inferir que, desde el punto de vista del tiempo en la jornada escolar diaria, un incremento del número de días lectivos, sin tener que aumentar el número de horas de la jornada escolar (pudiendo quedarse igual o incluso reducirla), puede ser una variable positiva a considerar para la mejora del rendimiento académico. No obstante, se trata de un factor más a considerar cuya mejora en los resultados (tras las modificaciones) estaría vinculado a una infinidad de variables interdependientes que afectarían de manera directa a esta interpretación y propuesta (nivel de atención sostenida del alumnado, curriculum escolar, metodologías educativas, contexto y realidad sociocultural, nivel y formación del profesorado, gastos en educación, etc.).

\section{Conclusiones}

Tras haber analizado la variable tiempo escolar en los países que han conformado nuestra muestra objeto de estudio, hemos podido comprobar la incidencia que puede tener su distribución y utilización en el rendimiento académico de los alumnos, pasando a comentar algunas ideas finales en torno al factor trabajado.

Respecto a las horas de instrucción obligatoria, podemos ver cómo el simple incremento de la carga horaria no repercute positivamente en el rendimiento académico. Países con mayor número de horas no obtenían mejores resultados en PISA, sino que, por el contrario, el rendimiento estaba vinculado a casos como el de Finlandia y Austria con una duración menor respecto al global de horas escolares durante la etapa obligatoria en primaria y secundaria. De esta manera, la calidad no está vinculada directamente con el número de horas sino con lo que se hace durante ese tiempo escolar.

No obstante, si podemos apuntar la idea de que reducir las jornadas lectivas puede beneficiar al proceso educativo y a la obtención de resultados positivos como ha quedado reflejado con los datos. Debido a ello, en el caso que desde el sistema educativo del país se quisiera modificar la distribución horaria de instrucción obligatoria (aumento, disminución o simple redistribución), los análisis comparativos muestran que es más positivo aumentar los días de asistencia al centro escolar que la duración de la jornada escolar, a tenor de los datos analizados.

Somos conscientes de que hay otras variables y factores que tienen una incidencia clave en el rendimiento académico de los educandos como la capacidad de atención, el curriculum escolar o las metodologías educativas que se desarrollen en el proceso de enseñanza-aprendizaje de cada uno de los países. Entre las mismas, destacamos algunas premisas que pueden repercutir positivamente en los resultados educativos:

- Ratio profesor-alumno: un menor número de alumnos por docente mejora la atención personalizada a los discentes, por lo que una menor ratio puede beneficiar a la calidad del proceso educativo (Barner, Carolan, Squires y Clarke, 2013). 
- Formación docente: a mayor nivel de preparación y formación del docente (por profesionales altamente cualificados y una óptima preparación y experiencia), mejor rendimiento puede obtener de sus alumnos.

- Gasto del país (PIB) en Educación: mayores partidas de recursos económicos permiten una mayor disponibilidad de medios y programas educativos innovadores.

- Organización curricular, pedagógica y metodológica: debemos elaborar programas educativos eficaces, estimulantes, adaptados a las necesidades correspondientes y que potencien las cualidades individuales del alumnado.

Por último, respecto a la variable tiempo escolar y su influencia en la calidad educativa como protagonistas de nuestro trabajo, queremos subrayar de nuevo la idea de que lo más determinante no es únicamente cuánto tiempo empleamos en la educación, sino que lo relevante es qué hacemos durante esos períodos lectivos para favorecer el desarrollo integral de nuestro alumnado.

No olvidemos que el tiempo es vida, por lo que las horas que los niños pasen en la institución escolar deben permitirles aprender el arte de vivir (Esteve, 2010) para que puedan desempeñarse con autonomía y eficacia en su contexto y realidad.

\section{Referencias bibliográficas}

Barnett, W.S., Carolan, M.E., Squires, J.H., Clarke Brown, K. (2013). The state of preschool 2013: State preschool yearbook. New Brunswick, NJ: National Institute for Early Education Research. Recuperado de: http://nieer.org/sites/nieer/files/yearbook2013.pdf

Bottani, N. (2006). La más bella del reino: el mundo de la educación en alerta con la llegada de un príncipe encantador. Revista de Educación, Extra 1, 75-90.

Bray, M. y Lai, K. (2010). La comparación de sistemas. En M. Bray, B. Adamson, y M. Mason (Coords.). Educación Comparada. Enfoques y métodos (pp. 159-184). Buenos Aires-México-Santiago-Montevideo: Granica.

Bruns, B. y Luque, J. (2014). Profesores excelentes: cómo mejorar el aprendizaje en América Latina. Washington: Banco Mundial. doi:10.1596/ 978-1-4648-0151-8

Colomo, E. (2013). Análisis de los valores presentes en las letras de las canciones con más impacto en 2010. Málaga: Spicum.

Colomo, E. (2015). ¿Cómo se educa para ser feliz? El papel del maestro en el desarrollo humano. Cuestiones Pedagógicas, 24, 59-74

Esteve, J.M. (2010). Educar: un compromiso con memoria. Barcelona: Octaedro.

Fernández, A.B. (2008). El tiempo en la clase de educación física: la competencia docente tiempo. Deporte y actividad física para todos, 4, 102-120.

Froemel, J.E. (2006). Los estudios internacionales del rendimiento y los países en vías de desarrollo: participación, resultados y relevancia. Revista de Educación, Extra 1, 131-152. 
Gabarda, V. (2015). Uso de las tic en el profesorado europeo, ¿una cuestión de equipamiento y formación? Revista Española de Educación Comparada, 26, 153-170.

Hernández, F. (2006). El informe PISA: una oportunidad para replantear el sentido de aprender en la escuela secundaria. Revista de Educación, Extra 1, 357-379.

Jabonero, M. (2015). La educación en América latina: apostando por el desarrollo de la equidad y la competitividad. En M. Jabonero, M. Martín Bris, A. Martínez Arias y J.L. Bizelli (Eds.). Miradas diversas de la educación en Iberoamérica. Madrid: Universidad de Alcalá.

Lavy, V. (2015), Do Differences in Schools' Instruction Time Explain International Achievement Gaps? Evidence from Developed and Developing Countries. The Economic Journal, 125, F397-F424. doi: http://dx.doi.org/10.1111/ecoj.12233

Lázaro, L. (2012). La perspectiva social en la jornada escolar en la Unión Europea. Estudio comparado en Italia, Francia, Portugal y España. Revista Española de Educación Comparada, 19, 193-218.

Manzón, M. (2010). La comparación de espacios. En M. Bray, B. Adamson, y M. Mason (Coords.). Educación Comparada. Enfoques y métodos (pp. 117-158). Buenos AiresMéxico-Santiago-Montevideo: Granica

OECD (2013). Resultados de PISA 2012 en foco: lo que los alumnos saben a los 15 años de edad y lo que pueden hacer con lo que saben. Consultado el 24 de diciembre de 2015. http://www.oecd.org/pisa/keyfindings/PISA2012_Overview_ESP-FINAL.pdf

OECD (2015), Panorama de la educación 2014: Indicadores de la OCDE. Madrid: Fundación Santillana. doi: http://dx.doi.org/10.1787/eag-2014-es.

Oliveira, R. y Castioni, R. (2016). Reseña: Prats, E. (2013). La educación, una cuestión de estado. Una mirada a Europa. Barcelona: Edición Universidad de Barcelona. Práxis Educativa, 11 (2), 542-546. doi: 10.5212/PraxEduc.v.11i2.0015

Pérez Gómez, A.I. (1.998). La cultura escolar en la sociedad neoliberal. Madrid: Morata.

Prats, E. (2013). La educación, una cuestión de estado. Una mirada a Europa. Barcelona: Edición Universidad de Barcelona.

Roselló, P. (1963). La estructura de la educación comparada. Revista Española de Pedagogía, 81, 3-14.

Ruiz Román, C. (2004). Identidades transculturales: los procesos de construcción de identidad de los hijos de inmigrantes marroquíes en España. Tesis doctoral. Málaga: Spicum.

Sandin, M. P. (2003). Investigación cualitativa en educación: fundamentos y tradiciones. Madrid: Mc Graw Hill.

Savater, F. (1999). Las preguntas de la vida. Barcelona: Ariel. 
Schiefelbein, E., Vélez, E. y Valenzuela, J. (1997). Factores que Afectan el rendimiento Académico en la Educación Primaria. Revisión de la Literatura de América Latina y el Caribe. Washington DC: Banco Mundial.

Schleicher, A. (2006). Fundamentos y cuestiones políticas subyacentes al desarrollo de PISA. Revista de Educación, Extra 1, 21-43.

Turner, R. (2006). El programa internacional para la evaluación de los alumnos (PISA): una perspectiva general. Revista de Educación, Extra 1, 45-74.

Velaz de Medrano, C. (2006). Presentación: una visión integral de las evaluaciones del PISA (OCDE) con especial atención a la participación de España. Revista de Educación, Extra 1, 13-18.

Vexliard, A. (1970). Pedagogía Comparada, Métodos y Problemas. Buenos Aires: Kapelusz. 